\title{
Sequential changes in autophagy in diabetic cardiac fibrosis
}

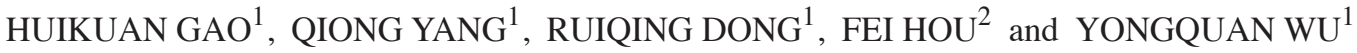 \\ Departments of ${ }^{1}$ Cardiology and ${ }^{2}$ Infection, Beijing Friendship Hospital, \\ Capital Medical University, Beijing 100050, P.R. China
}

Received December 12, 2014; Accepted October 2, 2015

DOI: $10.3892 / \mathrm{mmr} .2015 .4517$

\begin{abstract}
Autophagy is considered to be associated with cardiac fibrosis. However, whether autophagy accelerates or ameliorates fibrosis remains to be elucidated. In the present study, 36 rats were divided into two groups: Control rats and diabetic rats. The diabetic rats were established by feeding the animals a high fat diet combined with streptozotocin. From the two groups, six rats were sacrificed after 1, 6 and 7 months. Cardiac systolic functions were measured. The collagen volume fraction was calculated using Masson's trichome staining and the mRNA expression levels of type-I and type-III collagen were measured using reverse transcription-quantitative polymerase chain reaction (RT-qPCR) to assess the levels of cardiac fibrosis. The protein contents of microtubule-associated protein 1 light chain 3 (LC3) and sequestosome 1 (P62) were evaluated using western blotting, and the mRNA expression of Beclin 1 was measured using RT-qPCR, in order to assess autophagy. The results revealed that, in the diabetic rats, cardiac fibrosis developed and cardiac systolic function was reduced. In the hearts of the diabetic rats, the mRNA expression levels of collagen type I and III, and Beclin1 were upregulated; the ratio of the protein level of LC3-II/LC3-I was increased and the content of P62 was decreased. All the changes were aggravated as time increased. The changes in autophagy were correlated with those of cardiac fibrosis, suggesting that autophagy may have a synergistic role in diabetic cardiac fibrosis.
\end{abstract}

\section{Introduction}

Despite the fact that cardiac complications are major causes of mortality in patients with diabetes mellitus, therapeutic conceptions to prevent diabetic myocardiopathy remain inaccessible due to only partial understanding of the underlying mechanisms $(1,2)$. Diabetic cardiomyopathy was first recog-

Correspondence to: Professor Yongquan Wu, Department of Cardiology, Beijing Friendship Hospital, Capital Medical University, 95 Yong'an Road, Xicheng, Beijing 100050, P.R. China

E-mail:wuyongquan67@163.com

Key words: microtubule-associated protein 1 light chain 3, collagen, sequestosome 1, autophagy, cardiac fibrosis nized by Rubler et al in patients with diabetes (3). In the early 1980s, the observation was made that individuals with diabetes were able to develop cardiac fibrosis in the absence of other organic heart disease (4). This indicated that cardiac fibrosis may be important in cardiac complications of diabetes.

Autophagy, a highly conservative metabolic process in eukaryotic cells, is involved in cell growth and development, and in the maintenance of cell homeostasis, particularly under conditions of nutrient deficiency and stress $(5,6)$. However, excessive autophagy may contribute to cell death $(7,8)$. To date, a substantial number of reports have shown that autophagy is closely associated with the occurrence and development of several types of tissue fibrosis (9-11). However, whether autophagy accelerates or ameliorates fibrosis remains to be fully elucidated.

To investigate autophagy, cardiac fibrosis, and the correlations between the two, the present study used a combination of high-fat diet-fed and low-dose streptozotocin-treated rats to produce a model of type 2 diabetes (12). As it is known that diabetic patients without other heart diseases can develop cardiac fibrosis (4). The aim of the present study was to observe the sequential changes of autophagy in the process of cardiac fibrosis, and examine the correlation between autophagy and fibrosis.

\section{Materials and methods}

Ethics statement. All the rats used in the present study were raised in the Animal Experiment Center of Beijing Friendship Hospital, Capital Medical University (Beijing, China). The experimental protocol was approved by the Ethics Committee of Beijing Friendship Hospital, Capital Medical University.

Animal preparation. Male six-week old Sprague-Dawley rats were obtained from Vital River Laboratory Animal Technology Co., Ltd. (Beijing, China). The average weight of the rats was $200 \pm 20 \mathrm{~g}$, and they were housed with a $12 / 12 \mathrm{~h}$ light cycle, the temperature maintained between 21 and $25^{\circ} \mathrm{C}$, with free access to food and water. The rats were randomly divided into the following two groups (18 rats/group): Control rats (NC group) and diabetic rats (DM group). The control rats received a standardized diet (Beijing Ke'ao Xieli Feedstuff Co., Ltd., Beijing, China). The diabetic rats received a high-fat diet $(62 \%$ standardized diet, $15 \%$ fat, $3 \%$ egg yolk powder and $20 \%$ sugar $\%$ ) for 6 weeks. After 6 weeks, the control rats were administered with an intraperitoneal injection of vehicle 
solution ( $3 \mathrm{ml} / \mathrm{kg}$ body weight), comprising $0.1 \mathrm{~mol} / 1 \mathrm{citric}$ acid buffer (Yi Yan Sheng Hua, Shanghai, China), and were then provided with a standard diet. The diabetic rats were administered with an intraperitoneal injection of low-dose $1 \%$ streptozotocin (3 ml/kg body weight); Sigma-Aldrich, St. Louis, MO, USA), following which they were provided with a high-fat diet. The levels of blood glucose were measured using a glucometer (HEA-230; Omron, Osaka, Japan), with $0.2 \mathrm{ml}$ blood collected from the tail vein. All 18 rats in the diabetic group had a blood glucose level $>16.7 \mathrm{mmol} / 1$, which remained stable for 4 weeks thus were considered to be diabetic rats $(12,13)$. After 1,6 and 7 months, six rats from each group were anesthetized with an intraperitoneal injection of $10 \%$ chloral hydrate (30 mg/kg; Sigma-Aldrich), and the hearts were collected from the animals. Prior to sacrifice of the rats, animal echocardiography was performed.

Animal echocardiography. The echocardiography was performed following the rats being anesthetized with an intraperitoneal injection of $10 \%$ chloral hydrate $(30 \mathrm{mg} / \mathrm{kg}$; Sigma-Aldrich). The left ventricular ejection fraction (LVEF) and left ventricular fractional shortening (LVFS) were measured using a Vevo 770TM high-resolution microscopy ultrasonic system (Visual Sonics, Inc., Toronto, Canada).

Morphological assessment. The left ventricle of the rat hearts were excised and then fixed with $4 \%$ paraformaldehyde (Sigma-Aldrich). Paraffin sections $(1 \times 1 \mathrm{~cm})$ were produced using an embedding center (EG1130; Leica Microsystems GmbH, Wetzlar, Germany) and microtome (RM2235; Leica Microsystems $\mathrm{GmbH}$ ), then were stained with Masson's trichrome (Bestbio Company, Shanghai, China). Photomicrographs were obtained using the BX41 microscope (Olympus Corporation, Tokyo, Japan) and then quantified using Image-Pro Plus 6.0 Image software (Media Cybernetics, Inc., Rockville, MD, USA) to assess the percentage collagen volume fraction (CVF). The CVF (\%) was determined by calculating the density of the blue-stained area in the images over the total area.

Western blot analysis. Small sections $(100 \mathrm{mg})$ of the left ventricle of the rats were lysed with radioimmunoprecipitation assay buffer supplemented with a protease inhibitor cocktail from Roche Diagnostics (Summerville, NJ, USA). Protein was extracted and measured using a Bicinchoninic Acid (BCA) Protein Assay kit (CW Biotech, Beijing, China). Subsequently, the protein $(40 \mu \mathrm{g})$ was separated by $15 \%$ microtubule-associated protein 1 light chain 3 (LC3) or $10 \%$ sequestosome 1 (SQSTM1; P62) SDS-PAGE (CW Biotech) and were then transferred onto polyvinylidene fluoride membranes (Beijing Solarbio Science \& Technology Co., Ltd., Beijing, China). Subsequent to blocking with $5 \%$ milk for $2 \mathrm{~h}$ at room temperature, the membranes were incubated at $4{ }^{\circ} \mathrm{C}$ overnight with the following primary antibodies: Polyclonal rabbit anti-rat LC3B (ab62721; Abcam, Cambridge, UK) and polyclonal rabbit anti-rat P62 (P0067; Sigma-Aldrich), respectively. Following incubation, the membranes were washed and then incubated for $40 \mathrm{~min}$ at room temperature with horseradish peroxidase-conjugated secondary antibodies, followed by signal detection using an enhanced chemiluminescence detection system (GE Healthcare Life Sciences, Chalfont, UK).

Isolation of RNA and reverse transcription-quantitative polymerase chain reaction $(R T-q P C R)$ analysis. Total RNA was extracted from the tissues (100 $\mathrm{mg}$ ) using TRIzol reagent (Invitrogen; Thermo Fisher Scientific, Waltham, MA, USA), according to the manufacturer's protocol. The first cDNA strand was synthesized by reverse transcription reaction with a $20 \mu \mathrm{l}$ reaction mixture (Promega Corporation, MAdison, WI, USA) containing $4 \mu 1 \mathrm{MgCl}_{2}, 3 \mu \mathrm{l} 10 \mathrm{X}$ RT buffer, $2 \mu \mathrm{ldNTP}$ mixture, $0.5 \mu 1$ recombinant RNase inhibitor, 0.6 $\mu 1 \mathrm{AMV}$ reverse transcriptase, $1 \mu \mathrm{l}$ Oligo dT-Adaptor primer and $5 \mu \mathrm{g}$ RNA. The reverse transcription reaction product was then used for the amplification of cDNA with the reaction system using $10 \mu 1$ SYBR mastermix (Applied Biosystems; Thermo Fisher Scientific), $1 \mu 1$ upstream primer and $1 \mu$ l downstream primers, $1 \mu \mathrm{l} \mathrm{cDNA}$, and $7 \mu \mathrm{l}$ diethylpyrocarbonate-treated water (all fromPromega Corporation). qPCR was performed using a Mycycler thermal cycler (CFX96 Touch Deep Well; Bio-Rad Laboratories, Inc., Hercules, CA, USA) and analyzed using $2 \%$ agarose gels (Beijing Solarbio Science \& Technology Co., Ltd.). The following primer sequences from Changsha Guge Biotechnology Co., Ltd. (Beijing China) were used: Collagen type I, forward 5'-TGGTGAGACGTGGAAACCTG-3' and reverse 5'-CTTGGGTCCCTCGACTCCTA-3'; collagen type III, forward 5'-TTGGCACAGCAGTCCAATGTA-3' and reverse 5'-GGGCAGTCTAGTGGCTCATC-3'; Beclin1, forward 5'-GCTCTCGTCAAGGCGTCAC-3' and reverse 5'-CTTCAGCTACTTCCCGGTCG-3'; and GAPDH, forward 5'-GATGGGTGTGAACCACGAGAAA-3' and reverse 5'-ACGGATACATTGGGGGTAGGAA-3'. The PCR protocol was as follows: Denaturation at $95^{\circ} \mathrm{C}$ for $10 \mathrm{~min}, 40$ cycles of $95^{\circ} \mathrm{C}$ for $15 \mathrm{sec}, 64^{\circ} \mathrm{C}$ for $30 \mathrm{sec}, 95^{\circ} \mathrm{C}$ for $15 \mathrm{sec}, 60^{\circ} \mathrm{C}$ for $1 \mathrm{~min}$ and $95^{\circ} \mathrm{C}$ for $15 \mathrm{sec}$, and a final extension step of $60^{\circ} \mathrm{C}$ for $15 \mathrm{sec}$. The expression levels of target genes were determined following subtraction of the expression levels of GAPDH, with the value for control group designated as 1 , using the $2^{-\Delta \Delta \mathrm{Cq}}$ method.

Statistical analysis. Data are presented as the mean \pm standard error of the mean. Student's $t$-test was used for comparisons between two groups. Comparisons among three or more groups were analyzed using one-way analysis of variance. Pearson's product moment correlation coefficient was computed to determine the association between autophagy and fibrosis. $\mathrm{P}<0.05$ were considered to indicate a statistically significant difference. All statistical analyses were performed using SPSS 19.0 software (IBM SPSS, Armonk, NY, USA).

\section{Results}

Cardiac dysfunction is observed in the hearts of diabetic rats. The cardiac systolic functions of the rats were evaluated using echocardiography. After 1 month, no significant differences between the control rats and diabetic rats were observed. After 6 and 7 months, there were significant decreases in cardiac systolic function (LVEF and LVFS) in the diabetic rats, compared with the control rats. Systolic functions exhibited a decreasing trend over time in the diabetic rats; whereas no 
Table I. Left ventricular systolic function.

\begin{tabular}{lcccc}
\hline & \multicolumn{2}{c}{ Ejection fraction } & \multicolumn{2}{c}{ Fractional shortening } \\
\cline { 2 - 4 } Group & $\mathrm{NC}$ & $\mathrm{DM}$ & $\mathrm{NC}$ & $\mathrm{DM}$ \\
\hline 1 months & $77.38 \pm 6.99$ & $77.73 \pm 8.55$ & $47.22 \pm 6.24$ & $48.18 \pm 8.99$ \\
6 months & $85.53 \pm 3.12$ & $61.90 \pm 5.52^{\mathrm{a}, \mathrm{b}}$ & $55.92 \pm 3.65$ & $34.50 \pm 4.00^{\mathrm{a}, \mathrm{b}}$ \\
7 months & $80.25 \pm 7.38$ & $66.98 \pm 4.28^{\mathrm{a}, \mathrm{b}}$ & $53.74 \pm 6.31$ & $38.30 \pm 3.53^{\mathrm{a}, \mathrm{b}}$ \\
\hline
\end{tabular}

Data are presented as the mean \pm standard error of the mean ( $\mathrm{n}=6$ per group). ${ }^{\mathrm{a}} \mathrm{P}<0.05$, vs. $\mathrm{NC}$ at the same time; ${ }^{\mathrm{b}} \mathrm{P}<0.05$, vs. $\mathrm{DM}$ group at 1 month. NC, normal control rats; DM, diabetic rats.

A

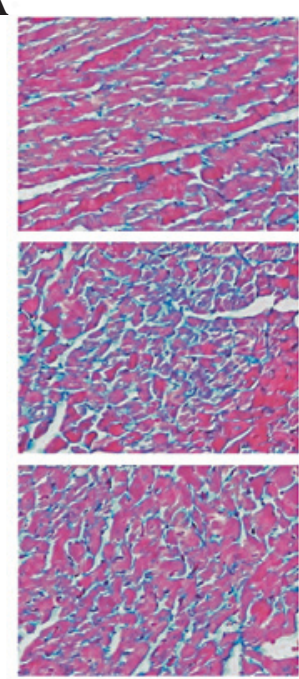

NC
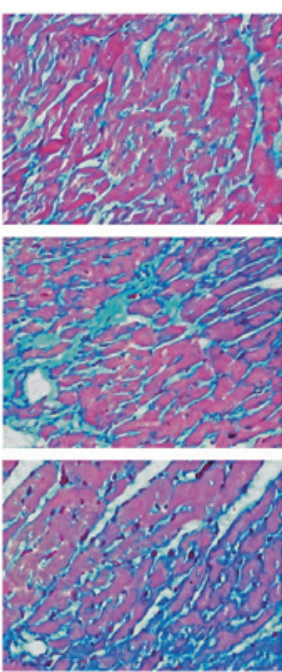

DM

\section{1 month}

6 month

7 month

B

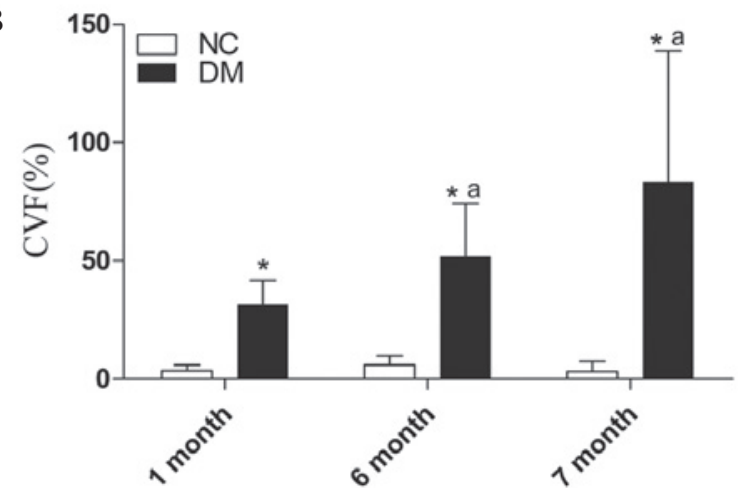

Figure 1. (A) Masson's trichrome staining was performed to evaluate the deposition of collagen in the left ventricular tissues. The heart tissues from the diabetic group exhibited focal regions of fibrosis (blue) in the interstitium. (B) CVF (\%) was determined by calculating the density of the blue area/total area. Magnification, $x 400$. Data are presented as the mean \pm standard error of the mean ( $\mathrm{n}=6$ for each group) ${ }^{*} \mathrm{P}<0.05$, vs. $\mathrm{NC}$ group at the same time; ${ }^{\mathrm{a}} \mathrm{P}<0.05$, vs. DM group at 1 month. NC, normal control rat; DM, diabetic rat. CVF, collagen volume fraction.

changes in systolic function were observed in the control rats (Table I).

Diabetes enhances interstitial collagen deposition. The results of the Masson's trichrome staining provided certain
A

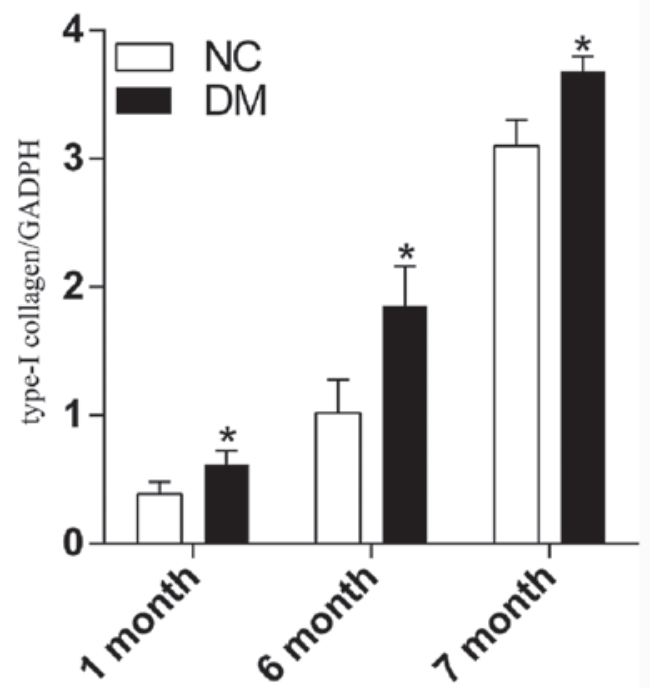

B

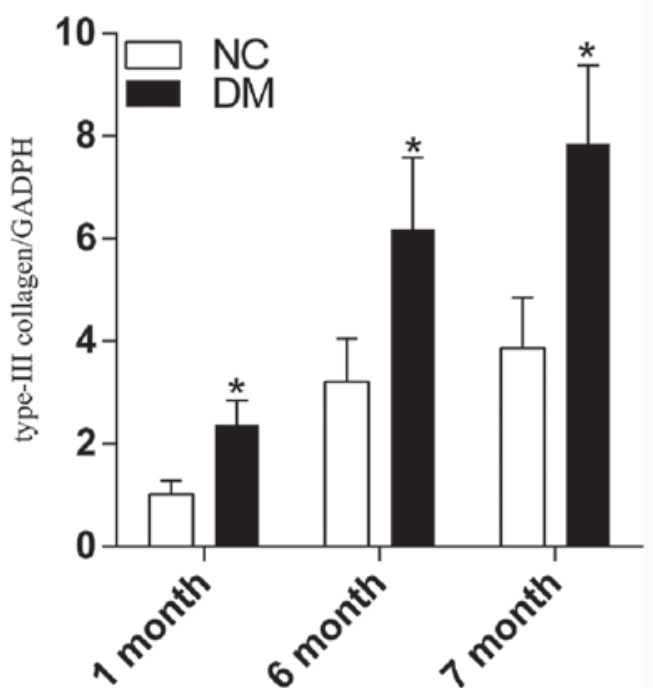

Figure 2. mRNA expression levels of (A) type-I and (B) type-III collagen were measured using reverse transcription-quantitative polymerase chain reaction analysis. The mRNA expression levels of type-I and type-III collagen were increased in the DM, compared with the NC rats. Data are presented as the mean \pm standard error of the mean ( $n=6$ per group). ${ }^{*} \mathrm{P}<0.05$, vs. NC group at the same time. NC, normal control rat; DM, diabetic rat.

clues regarding the levels of cardiac fibrosis in the rats. Representative patterns of Masson's staining demonstrating cardiac fibrosis are shown in Fig. 1A. The deposition of 


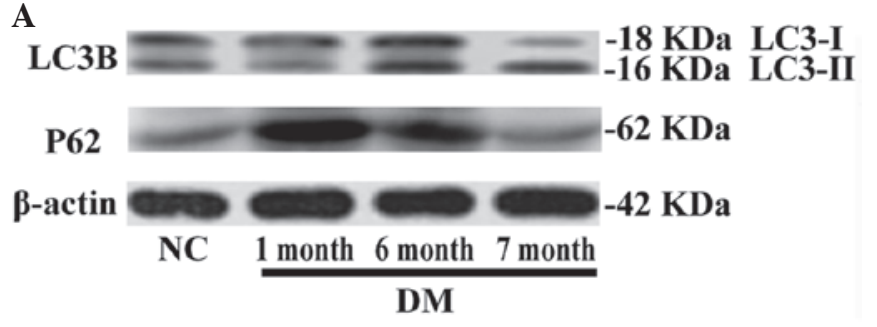

B

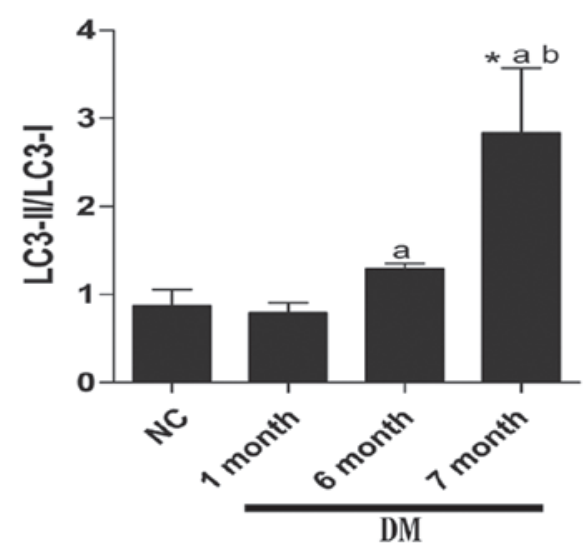

C

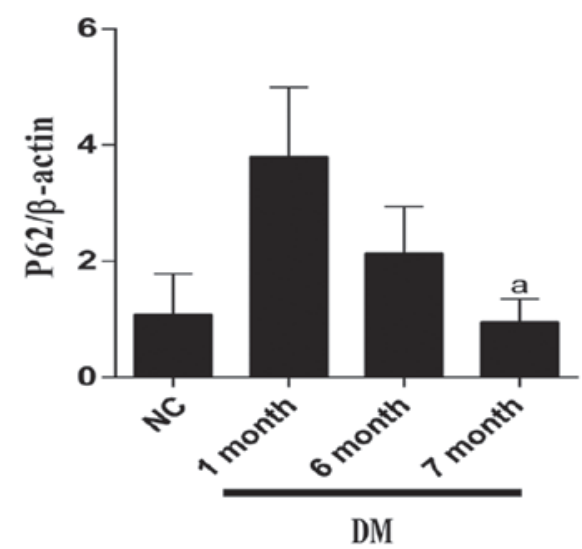

Figure 3. (A) Protein expression levels of LC3 and P62 were determined using western blotting. The ratio of the density of LC3-II/LC3-I, and the protein levels of P62 were normalized against the expression of $\beta$-actin $(\mathrm{n}=6)$. (B) Ratio of LC3-II/LC3-I was higher in the diabetic hearts over time. (C) Increase in autophagy was confirmed by the decrease in $\mathrm{P} 62 .{ }^{*} \mathrm{P}<0.05$, vs. $\mathrm{NC}$ group; ${ }^{\mathrm{a}} \mathrm{P}<0.05$, vs. DM group at 1 month; ${ }^{\mathrm{b}} \mathrm{P}<0.05$, vs. DM group of 6 months. LC3, microtubule-associated protein 1 light chain 3; P62 sequestosome 1; NC, normal control rat; DM, diabetic rat.

collagen in the myocardial interstitium of the heart tissues from the diabetic rats was significantly increased, compared with that observed in the heart tissues of the control rats. The collagen volume fraction exhibited an increase as time progressed in the diabetic rats; whereas no changes were observed in the control rats (Fig. 1B). Compared with the control rats, the mRNA expression levels of type-I and type-III collagen were significantly upregulated in the hearts from the diabetic rats (Fig. 2).

Expression levels of autophagy-associated proteins are upregulated in heart of diabetic rats. To assess the degree of autophagy in the rat heart tissues, following homogenization of the whole tissues, analysis was performed using western blotting (Fig. 3A) to examine the protein expression levels of

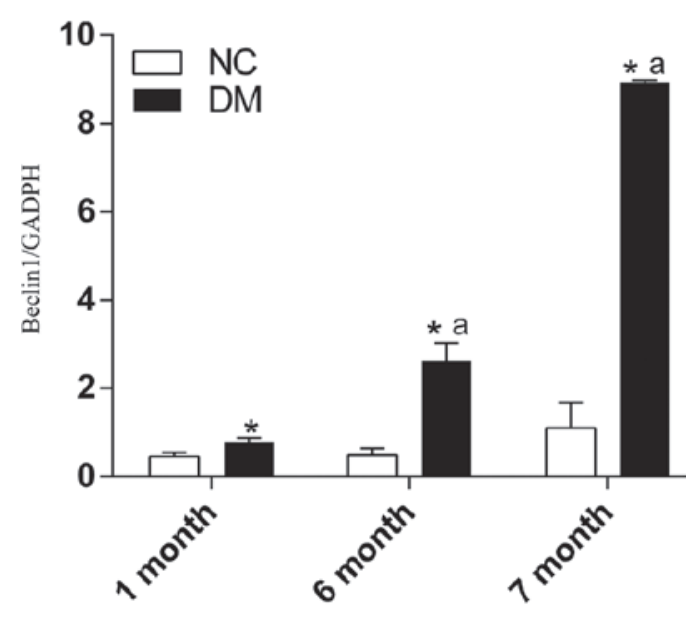

Figure 4. mRNA expression levels of Beclin1 in DM rats and NC rats were evaluated using reverse transcription-quantitative polymerase chain reaction analysis. Data are presented as the mean \pm standard error of the mean $(n=6)$. The mRNA expression of Beclin1 was increased in the DM rats compared with the NC rats. $(n=6) .{ }^{*} \mathrm{P}<0.05$ vs. $\mathrm{NC}$ group at the same time. ${ }^{\mathrm{a}} \mathrm{P}<0.05$, vs. DM group of 1 month. $\mathrm{NC}$, normal control rat; DM, diabetic rat.
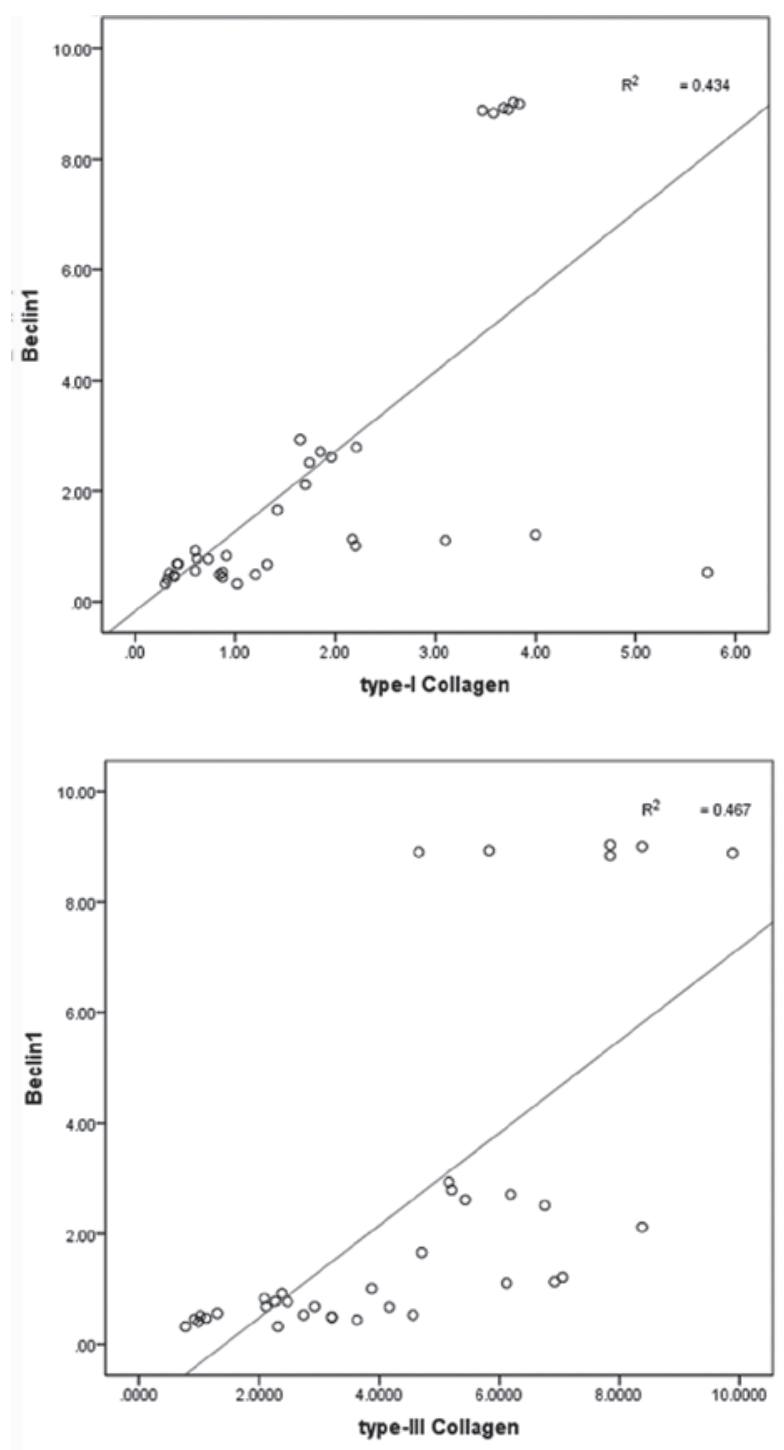

Figure 5. Positive correlation between the mRNA expression levels of Beclin1 with those of type-I and type-III collagen. $\mathrm{P}<0.05$. 
LC3-I and LC3-II, and to determine the ratio of LC3-II/LC3-I, which is a commonly used marker to determine the extent of autophagy (14). The results of the present study demonstrated that the hearts from the diabetic rats exhibited an increased ratio of LC3-II/LC3-I, compared with the ratio in the control rats(Fig. 3B), suggesting the upregulation of autophagosome formation. This upregulation increased over time, with the ratio after 7 months being significantly higher, compared with that in the control, and significantly higher than those after 1 and 6 months. The upregulation of autophagy was further indicated by the downregulation in the expression of P62 (Fig. 3C), which is known as an important measurement in evaluating autophagy pathway efficacy (15-17). In addition, the mRNA expression levels of Beclin1 were examined using RT-qPCR analysis. The mRNA expression level of Beclin1 was increased in the diabetic rats over time, compared with the control rats, which also indicated the upregulation of autophagy (Fig. 4).

Expression of Beclin1 is correlated with the expression levels of type-I and type-III collagen. To determine the correlation between the expression of autophagy-associated genes and cardiac fibrosis, Pearson's correlation coefficient was used to measure the similarity between the mRNA expression of Beclin1 and the expression levels of type-I or type-III collagen. The results revealed a positive correlation between the mRNA expression levels of Beclin1 with those of type-I and type-II collagen. (Fig. 5).

\section{Discussion}

It has been previously confirmed that abnormal cardiac function in patients with diabetes occurs at an early stage of insulin resistance, which indicates that cardiac fibrosis may have occurred prior to establishment of the clinical manifestations of diabetes (18). The formation of cardiac fibrosis in patients with diabetes is a slow and protracted process. Previous animal experiments have shown that the formation of a diabetic rat model of cardiac fibrosis requires at least 12-14 weeks, and that cardiac fibrosis is gradually aggravated as time progresses (19). Therefore, in the present study, 6 month and 7 month models of cardiac fibrosis were investigated, as cardiac fibrosis becomes more stable and more significant with increasing time. In the present study, the collagen volume fraction was calculated from Masson's trichrome staining, and the mRNA expression of collagen in the hearts from the diabetic rats exhibited notable increases. In addition, these increases were observed to be aggravated with increasing time. These findings are in agreement with the results of a previous study (20).

Increased cardiac fibrosis is a major factor responsible for myocardial stiffness and eventual systolic dysfunction (21). The present study demonstrated that the 6 month and 7 month diabetic rats exhibited a decrease in left ventrical systolic function. Of note, this decrease in left ventricle systolic function was not observed in the early diabetic heart, represented by the 1 month diabetic rats in the present study. Again, these results are consistent with those that have been previously reported (22).

Autophagy has been considered to provide a protective role in diabetic cardiac injury (2). The change of two important proteins/genes, LC3 and Beclin1, reflect the level of autophagy, particularly the ratio of LC3-II/LC3-I. When autophagy is activated, LC3 is conjugated to phosphatidylethanolamine, activating autophagy-related gene (Atg) 7, Atg10 and Atg4, leading to the conversion of LC3-I into LC3-II (23). This conversion is considered to be a marker of autophagy activity $(14,24)$. In the present study, it was found that autophagy was activated in the diabetic rats, and that the increase in autophagy was more marked with increasing duration. A previous study by Mellor et al (10) identified, in an animal model of diabetic mice reared on a fructose diet, that the peroxide produced by cardiomyocytes induces oxidative stress, which can activate autophagy, thereby inhibiting the proliferation of interstitial cells and interstitial fibrosis infiltration. The present study showed that, during the process of cardiac fibrosis, autophagic activity was gradually increased, and the change of autophagy was correlated with that of cardiac fibrosis, suggesting a potential synergistic role of autophagy in diabetic cardiac fibrosis.

In conclusion, the present study demonstrated the sequential change of autophagy in the process of diabetic cardiac fibrosis. The results of the present study indicate that autophagy may have a synergistic role in diabetic cardiac fibrosis. However, further investigations are warranted to elucidate the molecular mechanisms underlying autophagy in diabetes. This may assist in the development of novel therapeutic concepts for the treatment of diabetic cardiomyopathy (1).

\section{Acknowledgements}

This study was supported by funding from the ' 215 ' High-level Personnel Construction from Beijing Municipal Health Bureau, 215 Healthy and Technical Project (grant. no. 2011-3-003). The authors would like to thank Professor Likui Wang for revision of the manuscript.

\section{References}

1. Xu X, Kobayashi S, Chen K, Timm D, Volden P, Huang Y, Gulick J, Yue Z, Robbins J, Epstein PN and Liang Q: Diminished autophagy limits cardiac injury in mouse models of type 1 diabetes. J Biol Chem 288: 18077-18092, 2013.

2. Xie Z, Lau K, Eby B, Lozano P, He C, Pennington B, Li H, Rathi S, Dong Y, Tian R, et al: Improvement of cardiac functions by chronic metformin treatment is associated with enhanced cardiac autophagy in diabetic OVE26 mice. Diabetes 60: 1770-1778, 2011.

3. Rubler S, Dlugash J, Yuceoglu YZ, Kumral T, Branwood AW and Grishman A: New type of cardiomyopathy associated with diabetic glomerulosclerosis. Am J Cardiol 30: 595-602, 1972.

4. Fischer VW, Barner HB and Larose LS: Pathomorphologic aspects of muscular tissue in diabetes mellitus. Hum Pathol 15: 1127-1136, 1984

5. Zhu H, Tannous P, Johnstone JL, Kong Y, Shelton JM, Richardson JA, Le V, Levine B, Rothermel BA and Hill JA: Cardiac autophagy is a maladaptive response to hemodynamic stress. J Clin Invest 117: 1782-1793, 2007.

6. Onodera J and Ohsumi Y: Autophagy is required for maintenance of amino acid levels and protein synthesis under nitrogen starvation. J Biol Chem 280: 31582-31586, 2005.

7. Dorn GW II: Apoptotic and non-apoptotic programmed cardiomyocyte death in ventricular remodelling. Cardiovasc Res 81: 465-473, 2009.

8. Shen S, Kepp O and Kroemer G: The end of autophagic cell death? Autophagy 8: 1-3, 2012.

9. Hernández-Gea V, Ghiassi-Nejad Z, Rozenfeld R, Gordon R, Fiel MI, Yue Z, Czaja MJ and Friedman SL: Autophagy releases lipid that promotes fibrogenesis by activated hepatic stellate cells in mice and in human tissues. Gastroenterology 142: 938-946, 2012. 
10. Mellor KM, Bell JR, Young MJ, Ritchie RH and Delbridge LM Myocardial autophagy activation and suppressed survival signaling is associated with insulin resistance in fructose-fed mice. J Mol Cell Cardiol 50: 1035-1043, 2011.

11. Nho RS and Hergert P: IPF fibroblasts are desensitized to type I collagen matrix-induced cell death by suppressing low autophagy via aberrant Akt/mTOR kinases. PLoS One 9: e94616, 2014.

12. Srinivasan K, Viswanad B, Asrat L, Kaul CL and Ramarao P: Combination of high-fat diet-fed and low-dose streptozotocin-treated rat: A model for type 2 diabetes and pharmacological screening. Pharmacol Res 52: 313-320, 2005.

13. Islam MS and Choi H: Nongenetic model of type 2 diabetes: A comparative study. Pharmacology 79: 243-249, 2007.

14. Barth S, Glick D and Macleod KF: Autophagy: Assays and artifacts. J Pathol 221: 117-124, 2010.

15. Bjorkoy G, Lamark T, Brech A, Outzen H, Perander M, Overvatn A, Stenmark H and Johansen T: p62/SQSTM1 forms protein aggregates degraded by autophagy and has a protective effect on huntingtin-induced cell death. J Cell Biol 171: 603-614, 2005.

16. Lee HS, Daniels BH, Salas E, Bollen AW, Debnath J and Margeta M: Clinical utility of LC3 and p62 immunohistochemistry in diagnosis of drug-induced autophagic vacuolar myopathies: A case-control study. PLoS One 7: e36221, 2012.

17. Settembre C, Fraldi A, Jahreiss L, Spampanato C, Venturi C, Medina D, de Pablo R, Tacchetti C, Rubinsztein DC and Ballabio A: A block of autophagy in lysosomal storage disorders. Hum Mol Genet 17: 119-129, 2008.
18. Mizushige K, Yao L, Noma T, Kiyomoto H, Yu Y, Hosomi N, Ohmori $\mathrm{K}$ and Matsuo $\mathrm{H}$ : Alteration in left ventricular diastolic filling and accumulation of myocardial collagen at insulin-resistant prediabetic stage of a type II diabetic rat model. Circulation 101: 899-907, 2000.

19. Vadlamudi RV, Rodgers RL and McNeill JH: The effect of chronic alloxan- and streptozotocin-induced diabetes on isolated rat heart performance. Can J Physiol Pharmacol 60: 902-911, 1982.

20. Kato T, Yamashita T, Sekiguchi A, Sagara K, Takamura M, Takata S, Kaneko S, Aizawa T and Fu LT: What are arrhythmogenic substrates in diabetic rat atria? J Cardiovasc Electrophysiol 17: 890-894, 2006.

21. Boyle AJ, Shih H, Hwang J, Ye J, Lee B, Zhang Y, Kwon D, Jun K, Zheng D, Sievers R, et al: Cardiomyopathy of aging in the mammalian heart is characterized by myocardial hypertrophy, fibrosis and a predisposition towards cardiomyocyte apoptosis and autophagy. Exp Gerontol 46: 549-559, 2011.

22. Miyata S, Takemura G, Kawase Y, Li Y, Okada H, Maruyama R, Ushikoshi H, Esaki M, Kanamori H, Li L, et al: Autophagic cardiomyocyte death in cardiomyopathic hamsters and its prevention by granulocyte colony-stimulating factor. Am J Pathol 168: 386-397, 2006.

23. Zhang TZ, Fan B, Chen X, Wang WJ, Jiao YY, Su GF and Li GY: Suppressing autophagy protects photoreceptor cells from light-induced injury. Biochem Biophys Res Commun 450: 966-972, 2014.

24. Mizushima N and Yoshimori T: How to interpret LC3 immunoblotting. Autophagy 3: 542-545, 2007. 